\title{
Astroecology, cosmo-ecology, and the future of life
}

\author{
Michael N. Mautner* \\ Department of Chemistry, Virginia Commonwealth University, 1001 West Main Street, Richmond, VA 23284-2006, USA
}

\begin{abstract}
Astroecology concerns the relations between life and space resources, and cosmo-ecology extrapolates these relations to cosmological scales. Experimental astroecology can quantify the amounts of life that can be derived from space resources. For this purpose, soluble carbon and electrolyte nutrients were measured in asteroid/meteorite materials. Microorganisms and plant cultures were observed to grow on these materials, whose fertilities are similar to productive agricultural soils. Based on measured nutrient contents, the $10^{22} \mathrm{~kg}$ carbonaceous asteroids can yield $10^{18} \mathrm{~kg}$ biomass with $\mathrm{N}$ and $\mathrm{P}$ as limiting nutrients (compared with the estimated $10^{15} \mathrm{~kg}$ biomass on Earth). These data quantify the amounts of life that can be derived from asteroids in terms of time-integrated biomass $\left[B I O T A_{\text {int }}=\right.$ biomass $(\mathrm{kg}) \times$ lifetime (years) $]$, as $10^{27} \mathrm{~kg}$-years during the next billion years of the Solar System (a thousand times the $10^{24} \mathrm{~kg}$-years to date). The $10^{26} \mathrm{~kg}$ cometary materials can yield biota 10000 times still larger. In the galaxy, potential future life can be estimated based on stellar luminosities. For example, the Sun will develop into a white dwarf star whose $10^{15} \mathrm{~W}$ luminosity can sustain a BIOTA int $_{\text {of }} 10^{34} \mathrm{~kg}$-years over $10^{20}$ years. The $10^{12}$ main sequence and white and red dwarf stars can sustain $10^{46} \mathrm{~kg}$-years of BIOTA int in the galaxy and $10^{57} \mathrm{~kg}$-years in the universe. Life has great potentials in space, but the probability of present extraterrestrial life may be incomputable because of biological and ecological complexities. However, we can establish and expand life in space with present technology, by seeding new young solar systems. Microbial representatives of our life-form can be launched by solar sails to new planetary systems, including extremophiles suited to diverse new environments, autotrophs and heterotrophs to continually form and recycle biomolecules, and simple multicellulars to jump-start higher evolution. These programs can be motivated by life-centered biotic ethics that seek to secure and propagate life. In space, life can develop immense populations and diverse new branches. Some may develop into intelligent species that can expand life further in the galaxy, giving our human endeavors a cosmic purpose.
\end{abstract}

Keywords: asteroids; astrobiology; astroecology; cosmo-ecology; life in space; nutrients; biotic ethics; in situ resources

\section{Introduction}

It is the basic human identity that we belong to life. Life is unique in nature, and for us it is precious, as we are all fundamentally united with our family of organic DNA/protein life. Life also stands out in its intricately complex structures, and because the laws of physics precisely allow life to exist.

Further, all life are united in the active pursuit of selfpropagation. Belonging to life then implies a human purpose to secure, expand and propagate life. Indeed, we seek a higher purpose to our existence, and filling the universe with life can give our human existence a cosmic purpose. This purpose is best achieved in space, where life can have an immense future. While life is fragile on Earth, it can be secure in multiple worlds in space [1-4].

\footnotetext{
* Corresponding author. Email: mmautner@vcu.edu
}

Handling Editor: Beata Zagórska-Marek
Astrobiology addresses the place of life in nature: its origins, prevalence and future, all of which depend on the interactions of life with its environment. Astroecology addresses these interactions, to answer and quantify some basic questions:

(i) Were plausible past environments conducive to the origins and early sustenance of microorganisms?

(ii) Can we quantify the probability that life arises in favorable environments?

(iii) What is then the probability that extraterrestrial life exists?

(iv) Can space resources support life, and if so, how much?

(v) Can life migrate in space, and what roles may humans play?

(vi) What is the future of life in the Solar System on astronomical time-scales, and in the galaxy on cosmological scales?

These questions have been speculated on since antiquity. However, they can be addressed now scientifically, and in some aspects quantitatively. 
For example, experimental astroecology tests of meteorites showed that similar materials in asteroids can support bacteria, algae, plant cultures and even shrimp hatchlings, with fertilities comparable to agricultural soils (Fig. 1). Measured bioavailable nutrients in these materials allow estimating the potential amounts of life (time-integrated biomass) that can be constructed in this and similar solar systems. Further, predicted future energy sources in space allow estimating the potential amounts of life in the galaxy on cosmological time-scales. These subjects will be reviewed in the present paper.

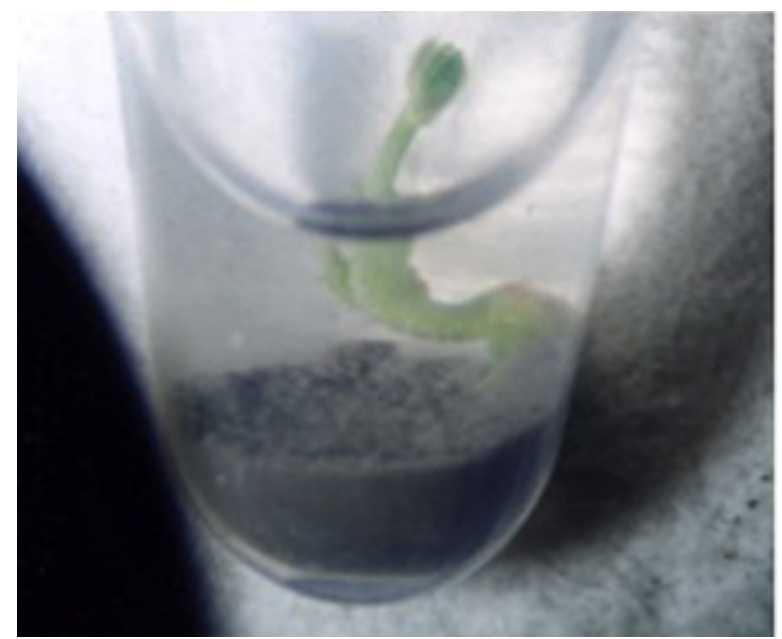

Fig. 1 Space is fertile: asparagus culture growing on meteorite soil [4].

\section{Astroecology}

\section{The definition, and a quantitative measure, of life}

Astroecology concerns the interactions of life with the space environment. For scientific purposes, it is necessary to define life and to measure or estimate its amounts quantitatively.

To estimate the amounts of life in ecosystems, "what is life?" must be first defined. In fact, defining life becomes a practical actionable question when we can control and alter life. If we aim to propagate life, we must define: what do we accept as fellow life that we seek to propagate?

Life can be defined broadly in terms of entropy and information [5]. However, our family of organic DNA/protein life focuses on propagating the species, which continues the biological, genetic, chemical patterns of life. At the heart of this process is the genetic code, which is used in translating genetic information encoded in nucleic acids into proteins. These proteins include enzymes that directly or indirectly help to reproduce the genetic code [6].

By this definition, life is a process. This process requires the flow of materials and energy, and uses information in molecular structures. Self-propagation is achieved by cycles in which DNA sequences are transcribed to RNA and then translated into proteins, including enzymes which help reproducing the DNA code, for example, by catalizing the biosynthesis of nucleic bases. All known biological cellular life, and only life, share these features.
We can therefore define life rationally: life is a process of active self-propagation by complex molecular structures through DNA/protein cycles.

\section{Quantifying life: biomass integrated over time active $\left(B / 0 T A_{\text {int }}\right)$}

For a scientific study of astroecology, life has to be quantified. A quantitative measure of life in an ecosystem can be formulated using the total amount of active biomass and its duration. This can be expressed in Equation (1) in terms of time-integrated biomass (biomass integrated over times available, $B I O T A_{\text {int }}$ ) measured in kg-years (similar to labor measured in men-years).

$$
\text { BIOTA }_{\text {int }}=\int m_{\text {biomass }, t} d t
$$

Here $m_{\text {biomass,t }}$ is the amount of biomass at time $t$ and integration is from time when life starts in the ecosystem to any given time $t$. Integration to the final inhabited time of the ecosystem, $t_{\mathrm{f}}$, yields the total amount of life in the ecosystem. This BIOTA ${ }_{\text {int }}$ may be measured in kg-years [7].

For a constant steady-state biomass BIOTA $A_{\text {steady-state }}$ (i.e., a constant biomass maintained by a balance of formation and destruction) lasting for time $\mathrm{t}$ in an ecosystem, the timeintegrated $B I O T A_{\text {int }}$ is then given simply by Equation (2).

$$
\text { BIOTA }_{\text {int }}=m_{\text {biomass,steady-state }} t
$$

For example, assuming that the present amount of life on Earth, on the order of $10^{15} \mathrm{~kg}$ [5] has been constant for the last billion years then BIOTA $_{\text {int }}$ on Earth has been $10^{15} \mathrm{~kg}$ $\times 10^{9}$ years $=10^{24} \mathrm{~kg}$-years. The potential life in the galaxy is immensely greater, on the order of $10^{48} \mathrm{~kg}$-years, and the potential life in the universe can be $10^{59} \mathrm{~kg}$-years [7].

Equation (2) yields BIOTA ${ }_{\text {int }}$ that can be derived from the resource materials. Since this depends on the duration of the biomass, it may be limited if wastage removes biomass irreversibly, such as by mineralization or leakage to space, as discussed below.

\section{Relations between resources and biomass}

The maximum possible amount of life in a finite ecosystem is defined by the amount of resource materials, their nutrient contents that can be used to construct biomass and the elemental requirements of the biomass.

The biomass that can be constructed from element $x$ in a resource material is given by Equation (3) [8,9].

$$
m_{x, \text { biomass }}=m_{\text {resource }} c_{x, \text { resource }} / c_{x, \text { biomass }}
$$

Here $m_{\text {resource }}$ is the mass of the resource material, $c_{\mathrm{x}, \text { resource }}$ and $c_{\mathrm{x} \text {,biomass }}$ are the concentrations of element $x$ in the resource materials and in biomass, respectively. Accordingly, $m_{\text {x,biomass }}(\mathrm{kg})$ of biomass could be constructed from $m_{\text {resource }}$ $(\mathrm{kg})$ of resource material if $x$ was the limiting element, i.e., the element in the resource materials that gives the smallest biomass. The limiting plant nutrients in nature, including meteorite soils, are usually bioavailable nitrogen $(\mathrm{N})$ or phosphorus (P).

For example, Tab. 1 shows the amounts of biomass that can be constructed based on several biologically key elements 
in carbonaceous chondrite meteorites. The yields vary among the various meteorite classes, with phosphate or nitrate as the limiting factors, while carbon and $\mathrm{K}, \mathrm{Ca}, \mathrm{Mg}$ and sulfate would allow larger biomass yields [7].

The previous sections quantify life in terms of active biomass. This does not account for the quality of life. However, a fraction of the biomass can support intelligent life. For example, presently about $10^{15} \mathrm{~kg}$ biomass on Earth [10] supports $7 \times 10^{9}$ people, requiring about $10^{5} \mathrm{~kg}$ supporting biomass per person. We assume below that in an efficient designed ecosystem $10^{4} \mathrm{~kg}$ biomass per person will be required.

Alternative to materials, energy may be the limiting factor. Equation (4) gives the biomass that a power source can sustain.

$$
m_{\text {biomass }}=P_{\text {source }} / P_{\text {biomass }}
$$

Here $m_{\text {biomass }}$ is the biomass sustained by the power source that outputs $P_{\text {source }}$ power (energy/time), while the biomass requires $P_{\text {biomass }}$ (power $/ \mathrm{kg}$ ) to function, including an efficiency factor. We consider below a requirement of $100 \mathrm{~W}$ per kilogram human biomass, i.e., $10 \mathrm{~kW}$ for a $100 \mathrm{~kg}$ human [4].

\section{Experimental astroecology}

\section{Meteorite models of asteroid soils}

Human settlement of the Solar System will require large scale in situ resource utilization. Settlements may be established in space colonies [3], on asteroids [11], on planets and their moons, including Mars [12,13]. These environments need to provide bioresources of organic carbon, inorganic plant nutrients, and water. The most accessible sources in the Solar System are found in carbonaceous $C$ type asteroids that contain these materials.

Can these materials really support life, and if so, how much? Fortunately, samples of the asteroids are available in carbonaceous chondrite meteorites, and samples of planetary resources in Martian meteorites. We measured their soluble, bioavalable contents of the nutrients shown in Tab. 1, and tested if soil microorganisms, colonizing cyanobacteria, algae, edible plants (asparagus and potato), and even small animals (brine shrimp) can grow on these space materials.
First, we tested the growth of microorganisms on meteorites. The results showed that a mixture of autotrophs (algae) and heterotrophs (fungi) can grow on meteorite and planetary materials, as required for a sustainable ecosystem that recycles nutrients (Fig. 2). The algal populations in the extracts were substantially larger than in the control deionized water, and approached populations reached in optimized BG11 nutrient medium (Fig. 3).
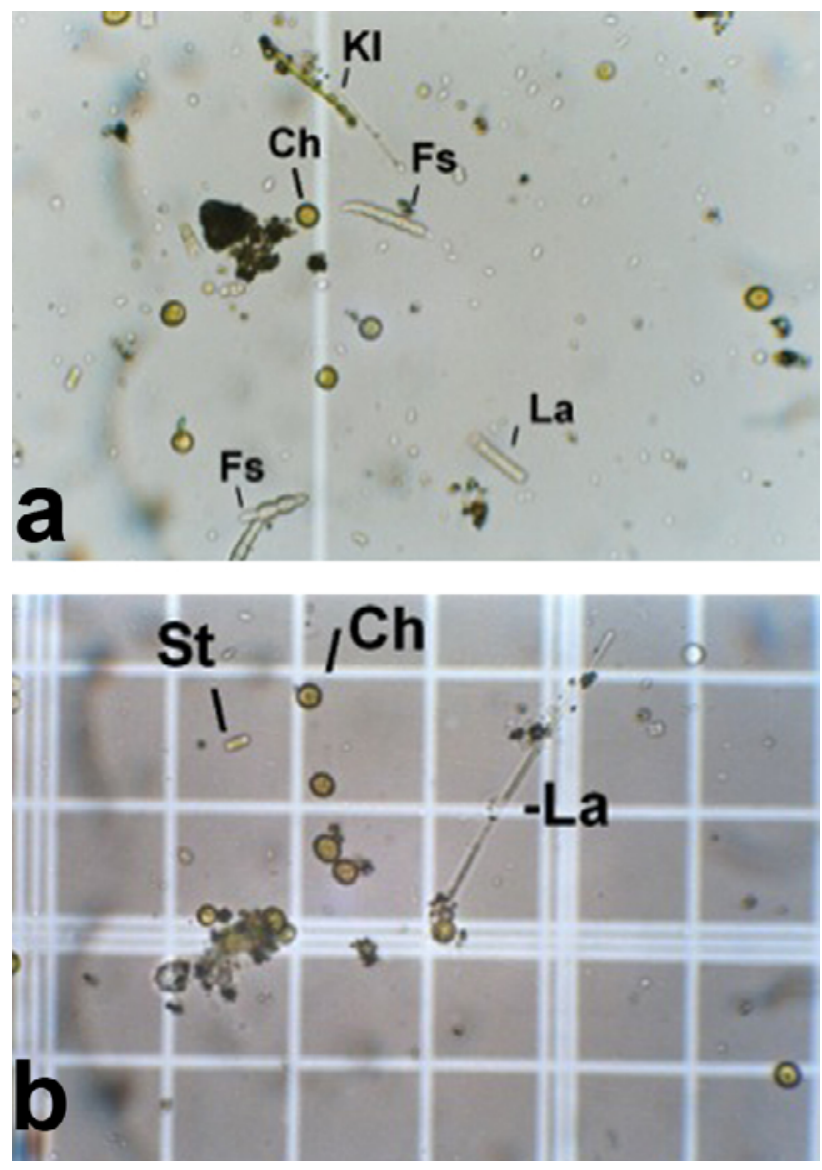

Fig. 2 Algal populations (colony forming units, $\mathrm{CFU} / \mathrm{ml}$ ) in extracts of Murchison carbonaceous chondrite (a) and Dar al Ghani 476 Martian meteorites (supplemented by 0.5 millimolar $\mathrm{NH}_{4} \mathrm{NO}_{3}$; b) after 32 days growth. $\mathrm{Ch}$ - Chlorella; $\mathrm{Kl}$ - Klebsormidium; La Leptolyngbya; St - Stichococcus; Fs - fungal spores [9].

Tab. 1 Biomass yields from water-soluble and total elemental contents in carbonaceous chondrite materials ${ }^{\mathrm{a}}$.

\begin{tabular}{|c|c|c|c|c|c|c|c|}
\hline \multirow[b]{2}{*}{ Meteorite } & \multirow[b]{2}{*}{ Type } & \multicolumn{6}{|c|}{ Biomass yield from element $(\mathrm{g} / \mathrm{kg})$} \\
\hline & & $\mathbf{C}$ & $\mathbf{N}$ & $\mathbf{K}^{+}$ & Nitrate-N & Phosphate-P & Sulfate- S \\
\hline Murchison & $\mathrm{CM} 2$ & 10.3 & 2.2 & 7.0 & 0.1 & 0.06 & 823 \\
\hline GRA 95229 & CR2 & 4.0 & 3.8 & 3.8 & 0.1 & 0.03 & 279 \\
\hline Allende & $\mathrm{CV} 3$ & 2.1 & 1.9 & 0.8 & 0.1 & 0.19 & 24 \\
\hline ALH 85002 & CK4 & 0.4 & 0.7 & 0.1 & 0.7 & 0.09 & 51 \\
\hline $\begin{array}{l}\text { Average biomass (from soluble } \\
\text { elements) }\end{array}$ & & 2.4 & 1.5 & 2.5 & 0.3 & 0.08 & 301 \\
\hline $\begin{array}{l}\text { Average biomass (from total } \\
\text { elements) }^{c}\end{array}$ & & 15.9 & 5.9 & 17.8 & --- & 74 & 2343 \\
\hline
\end{tabular}

${ }^{a}$ Units of g biomass/kg meteorite. Maximum biomass (g) of average composition that could be constructed from a given soluble element $x$ in $1 \mathrm{~kg}$ of each meteorite, if $x$ was the limiting nutrient. ${ }^{\mathrm{b}}$ Calculated using soluble contents from eight meteorites and average elemental concentrations in dry biomass. ${ }^{\mathrm{c}}$ As in ${ }^{\mathrm{b}}$, but total elemental contents [26]. 


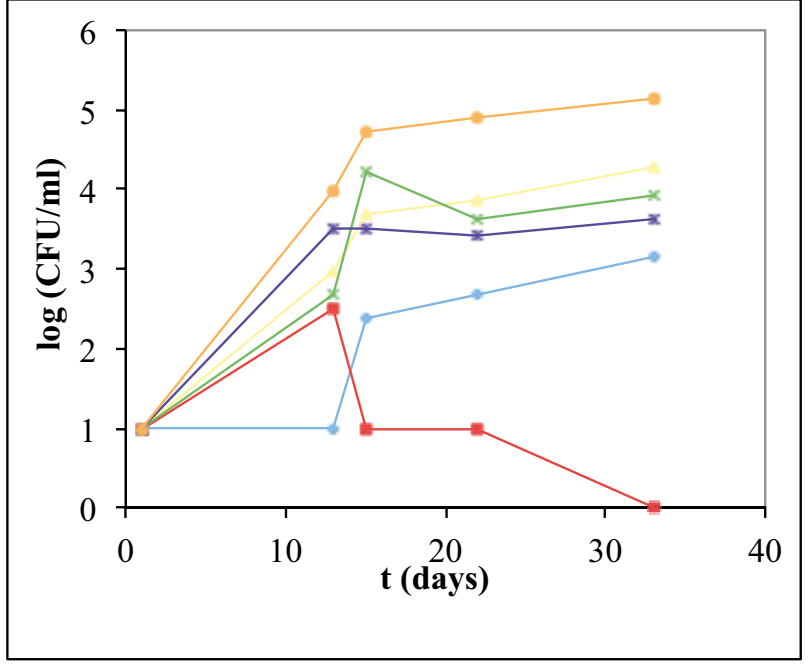

Fig. 3 Algal population growth in meteorite and simulant extracts and BG-11 nutrient medium. Allende (yellow line), Murchison (violet), DaG 476 (blue), Hawaii lava Mars simulant (green), BG-11 medium (orange), deionized water (red) [9].

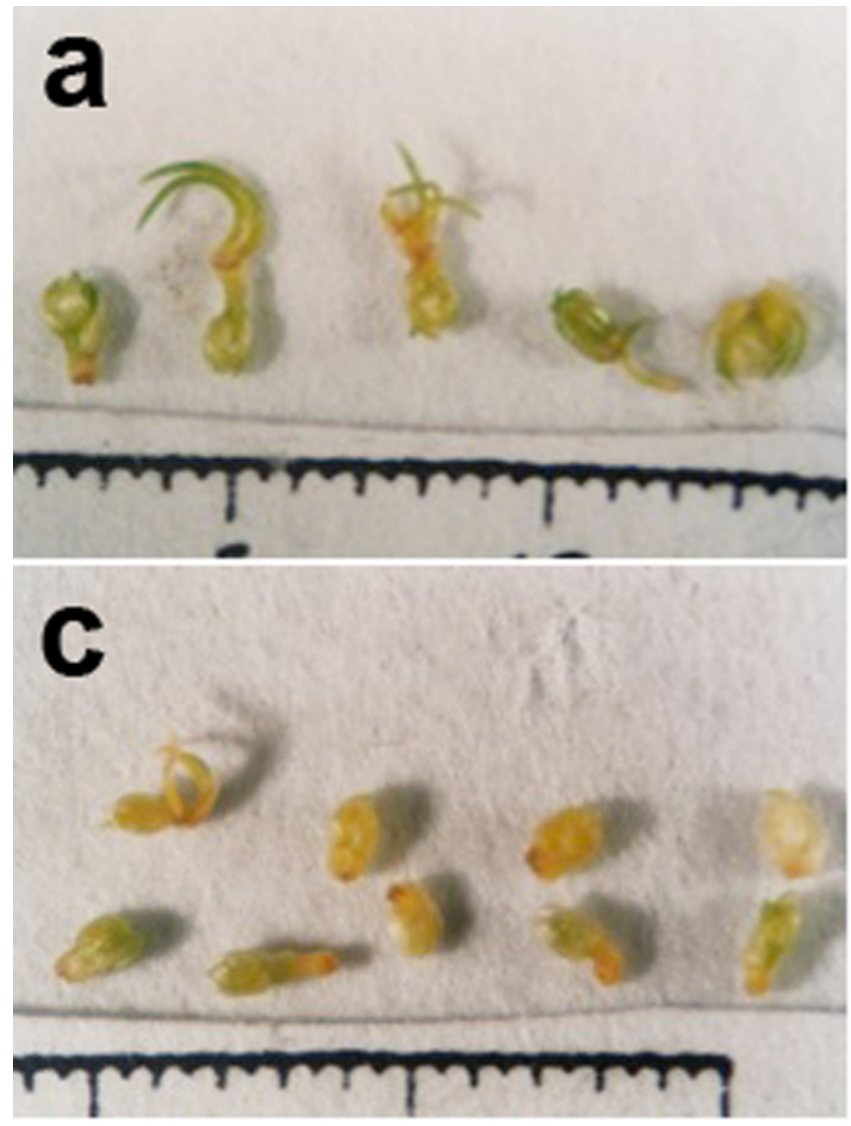

Further, asparagus cultures showed that nutrients from carbonaceous chondrite and Martian meteorites enhanced plant growth, especially the Martian meteorite because of its larger phosphate content (Fig. 4) $[8,9,14]$. We also found that brine shrimp eggs can uptake meteorite materials and can hatch in meteorite extracts, which shows that the extracts are not significantly toxic.

These experimental astroecology studies were followed by measuring the concentrations of soluble bioavailable carbon and nutrient electrolytes in carbonaceous chondrite meteorites. With these data, we calculated the potential biomass yields from several types of carbonaceous chondrites according to Equation (2) with $\mathrm{P}$ as the limiting nutrient [9]. Tab. 1 shows the calculated results for a composite soil with the average elemental contents of eight carbonaceous chondrite meteorites of various classes.

The measured bioavailable nutrient contents and the biological yields from asteroid/meteorite materials can be combined for rating their soil fertilities as compared with productive agricultural soils (Tab. 2).

The results showed that the carbonaceous chondrite meteorites have soil fertilities comparable to agricultural soils. Martian meteorites had the highest fertilities because of their high bioavailable phosphorus contents.

Further, these results allowed an experiment-based estimate of potential biomass in the Solar System that can be derived from asteroid resources, as discussed below.

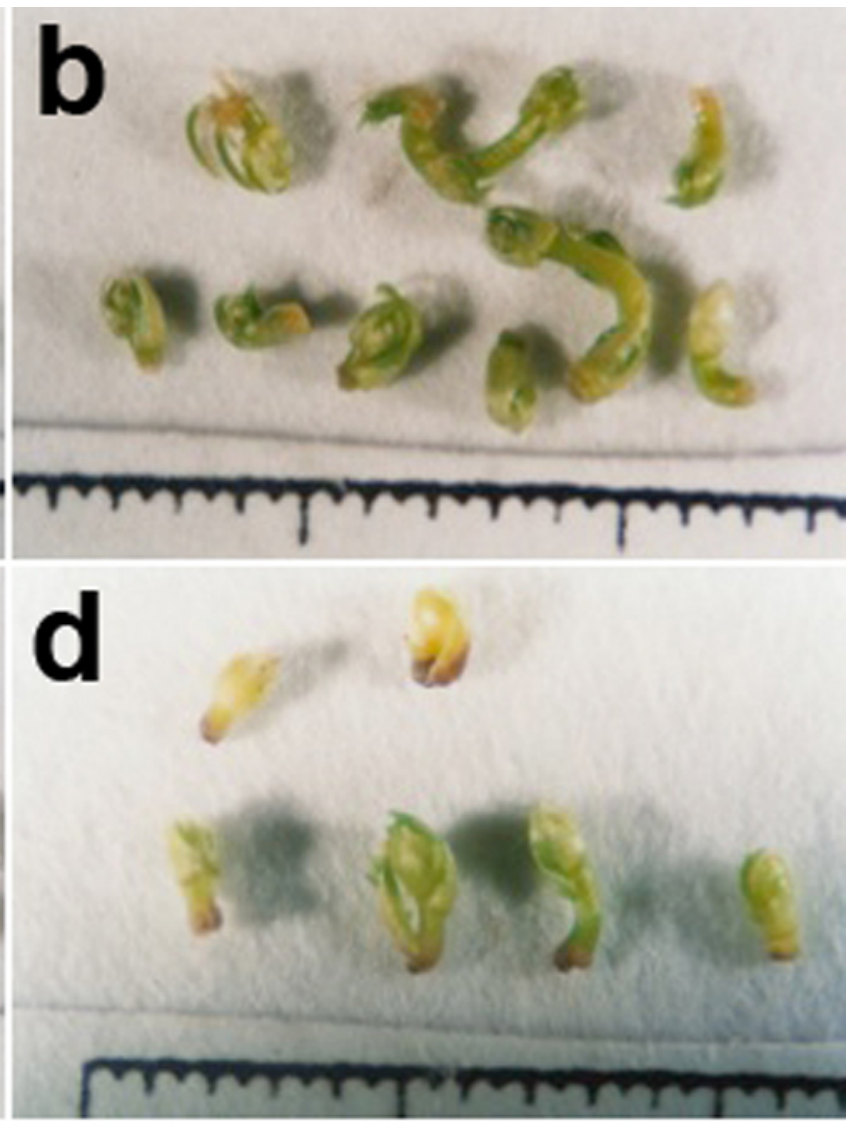

Fig. 4 Plant tissue cultures of Asparagus officinalis in meteorite and soil extracts, all supplemented by 5 millimol/l $\mathrm{NH}_{4} \mathrm{NO}_{3}$ and $3 \%$ sucrose. a Murchison CM2 meteorite. b DaG 476 Martian meteorite. c Water. d Hawaii lava Mars simulant. Scale divisions: small ticks $0.5 \mathrm{~mm}[9]$. 
Tab. 2 Fertility ratings of planetary materials according to biological yields and nutrient contents, and an overall fertility rating ${ }^{\mathrm{a}}$.

\begin{tabular}{lccccc} 
& Algal yield & $\begin{array}{c}\text { Average algal and } \\
\text { plant yield }\end{array}$ & N nutrient & P nutrient & Fertility rating \\
\hline Allende meteorite & + & ++ & + & + & Medium \\
Murchison meteorite & + & + & ++ & + & Medium \\
DaG 476 (Mars) & ++ & ++ & ++ & ++ & High \\
EETA 79001 (Mars) & +++ & 0 & 0 & +++ & Very High \\
Lunar simulant (lava ash) & 0 & ++ & 0 & ++ & Medium \\
Agricultural soil & ++ & ++ & + & High \\
\hline
\end{tabular}

${ }^{a}$ Ratings according to deviation from standard normal variate for each property: low $(0)$, medium $(+)$, high $(++)$, very high $(+++)$ yield or nutrient content [9].

\section{The human role}

\section{Expanding life in the Solar System: serving human needs and motivations}

The expansion of life in space can take dual routes: by human expansion in the Solar System, and by seeding new solar systems with microbial life. Interstellar human travel is desirable ultimately, but seems impractical with current levels of technology $[15,16]$.

Human expansion in the Solar System will depend on physical resources, technology, and on motivation. Concerning resources, the above astroecology experiments confirmed that asteroid materials can support life, and their measured nutrient contents allow estimating the supportable human populations.

Settling the Solar System can be motivated by serving human needs. These can include: satellite solar power stations for permanent clean energy [17]; a space sunshade against global warming $[18,19]$; mining of asteroids metals and structural materials $[11,20]$; large space colonies for growth and survival [3]; high resolution lunar telescopes [21]; and lunar gene banks for saving and re-cloning endangered species, including endangered human ethnic groups [22]. The space infrastructure that develops for these purposes will then allow exponential human growth in the Solar System, and serve as a base for seeding new solar systems [23-25].

The possible scope of space colonization can be based on Solar System resources that were estimated above. The resources and populations in various stages of space settlements, up to cities of millions, were examined recently [26].

On the long term, human expansion in space can be motivated by a responsibility to secure life. By necessity, we need to be the guardians of life, because only technological humans and post-humans can secure life to realize its immense potentials in space.

\section{Life in the Solar System: resources, biomass and populations}

Accessible resources are available in $\mathrm{C}$ type carbonaceous chondrite asteroids, and later in comets, that contain soluble bioavailable organics, electrolyte nutrients and extractable water. The bioavailable contents can be compared with the elemental requirements of biomass. This gives the potential yield of biomass from a unit mass $(\mathrm{kg})$ of resource materials
[Equation (3) and Tab. 1], with bioavailable $\mathrm{P}$ and $\mathrm{N}$ as the limiting elements $[8,9]$. For example, eight different carbonaceous chondrite meteorites contained an average bioavailable phosphate content of $c_{\mathrm{x} \text {,resource }}=0.0012 \mathrm{~g} / \mathrm{kg}$ meteorite solids [14]. In comparison, average dry biomass contains $c_{\mathrm{x}, \text { biomass }}$ $=15.5 \mathrm{~g} \mathrm{P} / \mathrm{kg}$ biomass [10]. Therefore the yield of biomass from this soil, $m_{\text {x,biomass }} / m_{\text {resource }}$ is $0.0012 / 15.5=7.8 \times 10^{-5}$ or roughly $10^{-4} \mathrm{~kg}$ biomass $/ \mathrm{kg}$ soil (Tab. 1 ).

On this basis, the estimated $10^{22} \mathrm{~kg}$ carbonaceous asteroids could yield $10^{18} \mathrm{~kg}$ biomass based on limiting P. If all of this was incorporated in human biomass, this could allow about $10^{16}$ humans.

By another estimate, in the Earth ecosystem $10^{15} \mathrm{~kg}$ biomass supports about $10^{10}$ humans, requiering $10^{5} \mathrm{~kg}$ sustaining biomass/human. A more efficient designed ecosystem may allow $10^{4} \mathrm{~kg}$ sustaining biomass/human. On this basis, the $10^{18} \mathrm{~kg}$ biomass derived from asteroid solubles could sustain a population of $10^{14}$ humans. In the next billion habitable years of the Solar System these would allow $10^{23}$ human-years and a total of $10^{27} \mathrm{~kg}$-years of time-integrated biomass, a thousand times more than the estimated $10^{24} \mathrm{~kg}$ time-integrated biomass on Earth to date.

\section{Human populations and the effects of wastage}

Equation (2) above yields the amount of biomass that can be derived from resource materials. However, the amount of life that is produced, in terms of time-integrated biomass BIOTA $_{\text {int }}$, also depends on the duration of the biomass. This may be limited if wastage removes biomass irreversibly. This wastage decreases the amount of biomass by a fraction $k_{\text {waste }}$ per unit time (year), and the remaining biomass after time $t$ is then given by $m_{\text {biomass, } \mathrm{t}}=m_{\text {biomass }, 0} \exp \left(-k_{\text {wastage }} t\right)$. Here $m_{\text {biomass }, 0}$ is the starting biomass and $m_{\text {biomass, } \mathrm{t}}$ is the remaining biomass after time $t$ [7].

From Equations (1) and (4) above follows a relation for the total time-integrated biomass BIOTA ${ }_{\text {int,total }}$ from time zero to infinity, for a biomass $m_{\text {biomass }, 0}$ formed from the resources and subject to wastage at the rate of $-k_{\text {wastage }} \times m_{\text {biomass,t }}(\mathrm{kg} /$ year $)$, giving $B I O T A_{\text {int,total }}=m_{\text {biomass }, 0} / k_{\text {wastage }}$. This relation applies to each unit of biomass that decays exponentially, regardless of when it was formed. The total integrated biomass BIOTA $_{\text {int,total }}$ depends only on the total amount of biomass created and its decay rate, but not on the rate of formation or on the 
biomass that exists at any time. Therefore, the lifetime of the ecosystem, yielding a given amount of life BIOTA $A_{\text {int,total }}$ can be extended by forming the biomass more slowly, and sustaining a smaller biomass longer.

A low $k_{\text {waste }}=0.01$, i.e., $1 \%$ per year of the steady-state biomass will be considered. With a steady-state biomass $m_{\text {biomass,steady-state }}(\mathrm{kg})$, then $k_{\text {waste }} m_{\text {biomass,steady-state }} t_{\text {ecosystem }}$ biomass is wasted during the lifetime of the ecosystem.

The amount of life in the ecosystem is maximized if all the resources are used during the lifetime of the ecosystem (here $10^{9}$ years). Considering $10^{18} \mathrm{~kg}$ biomass from soluble asteroid resources, the relation $k_{\text {waste }}(0.01) \times m_{\text {biomass,steady-state }}$ $\times t_{\text {ecosystem }}\left(10^{9}\right)$ years $=10^{18} \mathrm{~kg}$ allows maintaining a steady state biomass of $10^{11} \mathrm{~kg}$ by continually producing biomass to replace the wastage. This can support a population of $10^{7}$ (ten million) humans, during a billion future habitable years of the Solar System. With these parameters the asteroids then yield $10^{20} \mathrm{~kg}$-year time-integrated biomass supporting $10^{16}$ human-years in the Solar System.

If all the elemental contents of the asteroids can be used, this can produce a biomass larger by about a factor of 100 . Further, the $10^{26} \mathrm{~kg}$ comets, assuming compositions similar to asteroids, could support populations and biomass that are larger by a factor of 10000 than the above amounts based on the asteroids.

\section{Energy requirements}

Populations in space can use solar energy. The energy demand can be estimated based on an industrialized $1 \mathrm{~kW}$ power per person, plus $9 \mathrm{~kW}$ for the supporting biota, and a conversion efficiency of $10 \%$ of the collected solar energy to electricity, adding up to $100 \mathrm{~kW} /$ person (alternatively, if all the collected energy is used to support biomass, the power demand of $30 \mathrm{~W} / \mathrm{kg}$ biomass and $10^{4} \mathrm{~kg}$ supporting biomass per person would require collecting $300 \mathrm{~kW} /$ person). The above steady-state population of $10^{11}$ that was based on material resources then requires a power supply of $10^{16} \mathrm{~W}$, a small fraction of the $3.8 \times 10^{26} \mathrm{~W}$ output of the Sun.

Considering the power demands of the biomass, the estimated $10^{15} \mathrm{~kg}$ terrestrial biomass is supported by $3 \times 10^{16} \mathrm{~W}$ absorbed solar irradiance $\left(240 \mathrm{~W} / \mathrm{m}^{2}\right.$ absorbed irradiance $\times$ $1.3 \times 10^{14} \mathrm{~m}^{2}$ Earth cross section), i.e., $30 \mathrm{~W} / \mathrm{kg}$ biomass. At this rate, the above $10^{18} \mathrm{~kg}$ biomass derived from asteroids would require a solar power supply of $3 \times 10^{19} \mathrm{~W}$, still a small fraction of the solar output. Accordingly, the biota of the Solar System is limited by materials and not by energy.

Further in the future, the total elemental contents of the $10^{26} \mathrm{~kg}$ materials in comets, assuming a CM2 meteorite-like composition could yield a biomass of $10^{24} \mathrm{~kg}$ with a solar power demand of $3 \times 10^{25} \mathrm{~W}$, about $10 \%$ of the solar output, that could be collected by a Dyson sphere [27].

The above estimates concern the upper limits of biomass and populations in the Solar System about the main sequence Sun. Beyond that, life can continue after the Sun becomes a white dwarf star, and similarly about other future stars, on cosmological time-scales as discussed below. Life-centered ethics would recommend that as much of this potential life should be realized as possible, leading to great biological, social and intellectual advancements.

\section{The biology of human space adaptation}

The new planetary and space environments may present wide ranges of atmospheres, hydrospheres, geology, pressure, temperature, chemistry, $\mathrm{pH}$, lighting, radiation, and gravity. Some of these may have contributed to early life [28], and may require adaptation by future life. Further, the ultimate adaptation may be free living in open space. This may require new human features such as vacuum-tight containment; fully recycling self-contained metabolism; solar sail "wings"; asexual reproduction; hybrid algae/human organs for photosynthesis; organs for direct radio or laser communication; extended IR to UV vision; biological brains interconnected with computers; lifespans of centuries; social interactions among new life-forms; and psychological adaptations to extreme solitude or crowding.

For continuing our family of life, this transformed biota must still remain DNA/protein organic life. However, it can incorporate proteins with novel properties, using new amino acids, possibly coded by DNA that is extended with new nucleic bases [29], or other types of information-containing molecules. Moreover, to continue our genetic heritage similar to natural evolution, the genes of these human descendants should build on and incorporate human genes.

Artificial intelligence, durable robots or robot organs may be necessary in space. However, in silico "artificial life" is not life as defined commonly. Substituting ourselves with robots would eliminate, not propagate, our organic DNA/protein life-form. Even in space and through engineered evolution, the continuation of biological life is best assured through control by biological brains (as opposed to computers) with a vested interest to continue DNA/protein life.

The profound changes in biology can affect human nature itself, and even the core processes of biology. This may seem futuristic, but scientists are already developing these new biotechnologies [30]. These developments will affect the genetic heritage of all present humanity, and should be therefore subject to informed public approval.

The transformation of life will also require psychological and philosophical adaptations that promote survival. In fact, destruction (high entropy) is easier than survival, and any advanced society can self-destruct. Aggression then needs to be modified, and re-directed to motivate the expansion, not the destruction, of life. In a self-fulfilling future, the conscious pursuit of survival will be essential to secure survival.

\section{Early life and the origins of ecology}

\section{Chemical kinetics and the probability of life}

Life can exist and expand in the Solar System for its expected five billion habitable years, but this is a minute fraction of the possible future life in the universe.A main question is if life already exists in other Solar Systems, or if we are alone, with the future of life in our hands. If so, we can end our cosmic isolation by seeding new solar systems with life; and if we are alone, it is, by biotic ethics, a moral duty to do so.

The prevalence of life in the universe can be estimated by the probability that life arises and survives in favorable environments. There may be many other habitable solar systems 
throughout the galaxy, but the probability for life to start there may be very small, because all viable self-replicating life forms need complex interactive biological functions. The required components include all four nucleic bases and corresponding nucleosides and nucleotides, biological amino acids, ADP and ATP, membranes and mebrane transport apparatus. These components have millions of isomers and related structures, and the products of non-selective abiotic synthesis from simple compounds would have spread over millions of compounds, that would then all have too low concentrations to allow further synthetic reactions. Examples are the mixture of $\mathrm{D}$ and $\mathrm{L}$ isomers of biological and nonbiological amino acids in meteorites. Similarly, if all nucleic base and amino acid analogues and isomers were present equally, their concentrations would be too low for forming more complex biomolecules.

Even the first viable proto-cell needed these complex biochemical functions and apparatus. It would have had a very small probability to self-assemble through many steps with many possible outcomes, only a few of which lead to life.

Indeed, it is remarkable that a single-cell cyanobacterium can convert a few simple compounds (water, $\mathrm{CO}_{2}$, ammonia, phosphate) into thousands of complex molecules, which would be all required in a viable precursor. For example, the genome of cyanobacteria is in the range of $10^{6}-10^{7}$ base pairs $[31,32]$. Assuming that in a more simple protocell sequences of only a few hundred DNA or RNA bases coded peptides and proteins, even this simple genome could have still coded tens of thousands of different peptides and proteins, each of which catalyzed the synthesis of a specific biomolecule.

In turn, in an interactive system such as a protocell, every molecule may react with another molecule, including selfreactions. Considering bimolecular reactions, $\mathrm{n}$ compounds can then undergo $n^{2}$ different reactions. For example, 1000 compounds can undergo $10^{6}$ reactions, with rates determined by the pseudo-first order rate coefficients that are affected by the $n$ reactant concentrations and other variables such as temperature, pressure, $\mathrm{pH}$, ionic strength, homogenous catalysis by metal ions, heterogenous mineral catalysts, inhibitors, and IR to UV light intensities.

All of these parameters can have a continuum of values, but for simplicity, assume only ten distinct values for each. Then 10 physical parameters each with 10 discrete values allow $10^{10}$ different combinations affecting each of the $10^{6}$ reaction rate coefficients, for a total of $10^{16}$ different chemical states (where a chemical state is a combination of the parameters that define that state). This model is still oversimplified because every bimolecular reaction may be catalyzed or inhibited by each of 1000 chemicals in the cell. These termolecular interactions increase to $10^{19}$ the number of possible chemical states, and decrease the probability of a spontaneous assembly of a viable protocell to $10^{-19}$ accordingly. Further, the protocell had to arise in a supporting environment that also has many variables, which further decreases the probability to form a viable protocell.

Moreover, this first protocell needed to arise in a survivable environment, and the microbial population had to able to adapt to changing environments, but this involves more advanced biological capabilities.

\section{The origins of ecology}

A viable first cell must emerge in an environment where it can survive, multiply and evolve. The probability $P_{\text {biosphere }}$ that a viable biosphere arises may be expressed in a truncated Drake-type Equation (5) [33], where $P_{\text {origin }}$ is the probability that first life arises in a given environment, and $P_{\text {survival }}$ is the probability that this environment can sustain it.

$$
P_{\text {biosphere }}=P_{\text {origin }} P_{\text {survival }}
$$

For example, by the above estimates, the probability that a viable self-propagating system arises is $P_{\text {origin }}<10^{-19}$ in a sustaining environment where $P_{\text {survival }}<10^{-6}$ (one of $10^{6}$ possible states; Fig. 5), i.e., $P_{\text {biosphere }}<10^{-19} \times 10^{-6}=10^{-25}$.

Further, once a viable self-replicating multiplying protocell arises, its population can grow exponentially, and all the nutrients in the environment would become sequestered in this biomass. The continuing availability of nutrients and essential organics would require continuing biosynthesis (autotrophs) and recycling (heterotrophs), but such complex populations are unlikely in the first ecosystem.

Alternatively, the first cell would have to synthesize its biomolecules from simple compounds, but this already requires catalysis by complex biomolecules such as enzymes in the first place (the "chicken or eggs first" paradox).

In summary, even the first sustainable ecosystem must provide the continuing supply, production and recycling of biomass for a sustainable ecosystem. Fig. 5 summarizes this early ecology.

The active biomass can establish a steady-state involving the cycle in Fig. 5. The total mass of the system can achieve a steady state depending on the production rates of organics and on their depletion rates to form inactive end products.

Fig. 5 may be too complex for analytical solution but may be suitable for computer modelling. The amount of active biomass is controlled by six rate coefficients even in this simple scheme. If each can have only 10 distinct values, only one of which sustains life, then as in Equation (7), this ecological complexity alone would decrease the probability of a viable early ecosystem by a factor of one million.

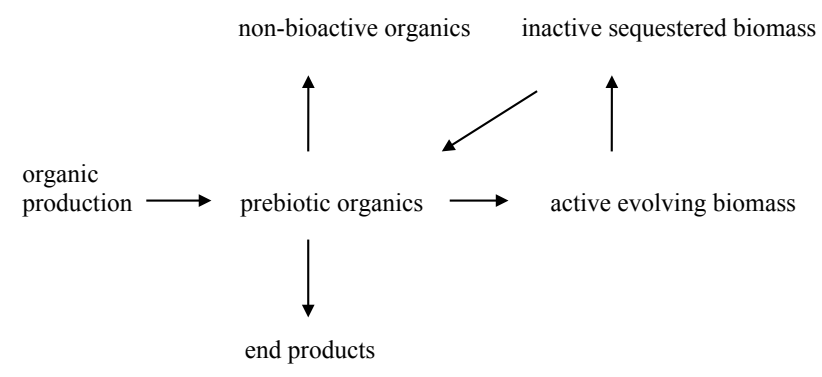

Fig. 5 Processes in an early ecosystems.

Further, once sequestered in biopolymers, these organics would not be available for further biosynthesis. Therefore, a continual supply of biomolecules is needed through photosynthesis by autotrophs and recycling by heterotrophs. A natural founding population of protocells is unlikely to 
be able to fulfil all of these functions, but directed seeding populations could do so. An alternative continuous source of organics may be the infall of interplanetary dust, meteorites and comets. The requirements of early ecosystems are discussed further below.

\section{Can the probability of life be tested experimentally?}

The earlier question: "can we quantify the probability of life?" can be restated: "can we compute or test experimentally the probability that life arises, given plausible source materials and environmental conditions?" A scientific model would have to explore all the chemical states of a plausible pre-biotic chemical system, i.e., all possible combination of the variables that affect the system.

For example, by the preceding estimates, a viable protosystem has to be in one of $10^{19}$ chemical states in an environment that can have one survivable set of parameters out of $10^{6}$ possible sets, i.e., $P_{\text {biosphere }}=10^{-19} \times 10^{-6}=10^{-25}$. An experimentally verifiable model would have to test experimentally these $10^{25}$ states.

If one of the $10^{25}$ states could be tested in one day for forming a viable protocell, then the experimental testing would require $10^{25}$ days or about $10^{18}$ human-years (a billion scientists working for a billion years) to test this simplified model, which is only one of practically innumerable possible prebiotic chemical systems with various compositions and physical parameters. Even this would yield only one of many possible mechanisms for life to arise and would not identify how life actually started. Apparently, a realistic experimental model for the probability to form a viable biological ecosystem stochastically from plausible precursor chemicals may not be feasible.

In other words, the origins of complex life involve an improbable coincidence of a very large number of physical and chemical variables. It seems unlikely that such a model can be constructed, much less tested experimentally to verify proposed mechanisms. It is then not possible to quantify the probability that life arose and now exists elsewhere. Without such information, and if astronomers don't find extraterrestrial (ET) life, we may need to consider that terrestrial life may be alone (extraterrestrial means here life outside our Solar System. Microorganisms on other planets in this Solar System, that result from material exchanges among planets such as in Martian meteorites, would be irrelevant to the probability of independent origins elsewhere. To distinguish ET life in and outside the Solar System, a compact terminology for solar system may be solys, and for life outside our Solar System, extrasolys life).

\section{Seeding the galaxy}

\section{An overview of directed panspermia}

With the existence or probability of extraterrestrial life unknown, we may be alone to secure the future of life. We can then make sure that life will continue and expand, by seeding new solar systems with representatives of our organic DNA/protein life-form. In fact, our biological unity with all life, combined with our technical abilities, imply a moral responsibility to secure and expand life in space.
The essential structures and processes of our cellular organic life are present in every cell from microorganisms to humans. To carry this basic information, microorganisms can be launched to space in large numbers to start new ecosystems that can lead to new species and a galacticscale biodiversity. There is some urgency, because we don't know how long our space-faring technology will endure. Interfering with indigenous life can be avoided by seeding newly forming young solar systems where local life could not have started yet.

Similarly, directed panspermia by another civilization could have started life on Earth after arising elsewhere [34]. Conversely, directed panspermia from Earth to space was also considered [35,36], and its scientific and ethical aspects have been developed in some detail [23-25,37-39]. From the new habitats, life may expand further by natural or directed panspermia, as an effective mechanism for the expansion of life.

Panspermia missions may aim to seed extrasolar planets, accretion disks about young stars, or star-forming zones in interstellar clouds. Each strategy has different requirements and probabilities of success.

Mature planets nearby, with liquid water, may be ready for colonizing microorganisms. These habitable extrasolar planets are small and hard to aim at accurately, but dispersing the microbial capsules in orbit can increase the probability of capture (Fig. 6) [23]. For example, for a reasonable probability of success we may send 100 capsules to each target, i.e., $n_{\text {(capsules) }}=100$, carrying 100000 microorganisms of $10^{-15}$ $\mathrm{kg}$ each and a biomass payload of $10^{-10} \mathrm{~kg}$, delivering a total of $10^{7}$ microorganisms to seed the planet.

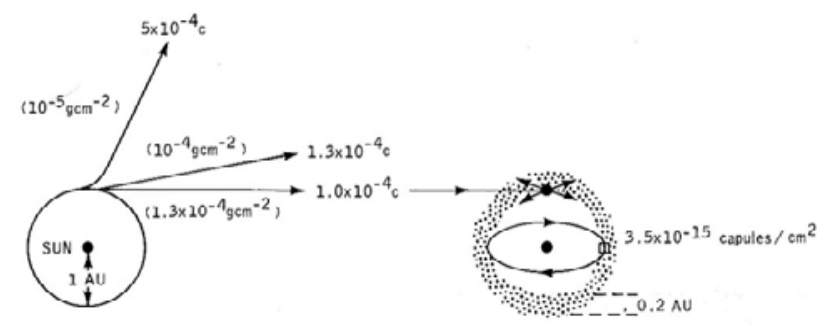

Fig. 6 Launch of microbial payloads from Earth orbit to target planetary systems by solar sail propulsion, and dispersion and capture at the extrasolar planet [23].

\section{Seeding star-forming clouds}

The preferred strategy could aim at star-forming interstellar clouds as the largest and easiest targets (Fig. 7). An important advantage is that local life, especially advanced intelligent life, would not have developed yet in these new solar systems, avoiding biological interference. Also, stars develop in clusters in these clouds and one mission can seed dozens of new solar systems [25].

One potential target is the Rho Ophiuchus cloud 520 lightyears (ly) away, that contains zones with various densities as they progress to star formation [40]. The missions may target the entire cloud; within these clouds, specific dense condensation cores; within these cores, protosolar condensations that form stars; and within these condensations, 


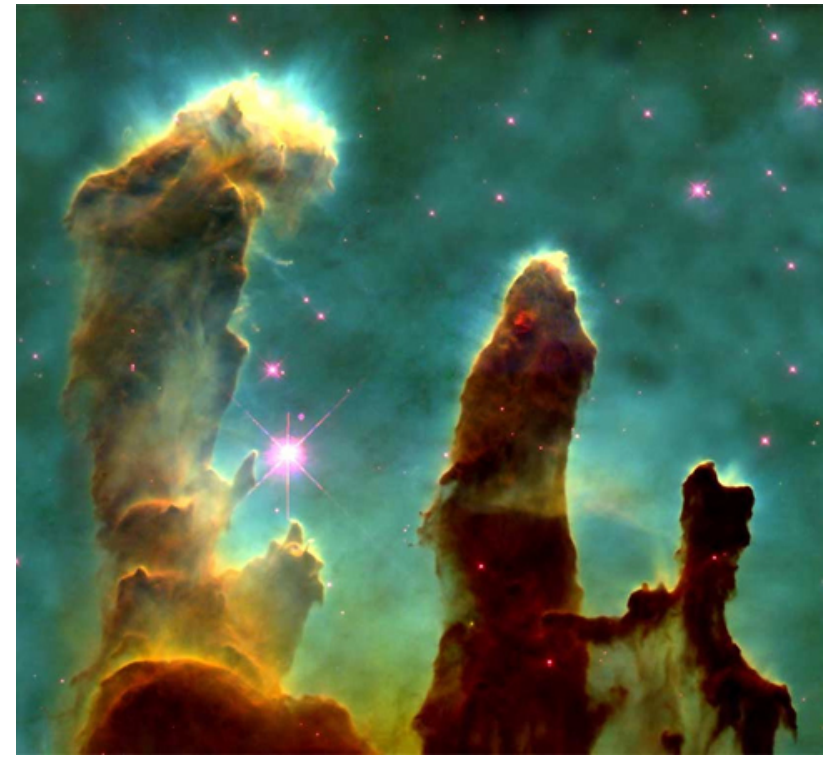

Fig. 7 Young stars in interstellar clouds (NASA Hubble Space Telecope). Panspermia missions can aim for the clouds, for dense star-forming zones within them, or for accreting planetary systems about the new stars (red dots).

accretion disks about new stars. It is desirable to target the smaller zones with high precision, where the capsules will mix with less dust and therefore a larger fraction will be then delivered to planets [25].

The probability $P_{\text {(target) }}$ that a mission will arrive at a target zone is given by Equation (6). Here $A$ is the area of the target, $\delta y$ is its positional uncertainty at the time of arrival, $r_{\text {(target) }}$ is the radius of the target zone, $v$ is the velocity of the vehicle, $\alpha_{\mathrm{p}}$ is the uncertainty of the angular proper motion of the target, and $d$ is the distance to the target.

$$
P_{(\text {target })}=\frac{A_{(\text {target })}}{\pi(\delta y)^{2}}=\frac{r_{(\text {target })}^{2} v^{2}}{\alpha_{p}^{2} d^{4}}
$$

The probability of arrival to the target can be increased by selecting larger targets $\left(r_{\text {(target) }}\right)$, increasing the velocity of travel (that decreases the positional uncertainty of the target at arrival), decreasing the uncertainty of position of the target (increasing precision of astrometry) and decreasing $d$ (closer targets). Equation (6) yields the $P_{\text {(target) }}$ values in Tab. 3. Values of $P_{\text {(target) }} \geq 1$ means unit probability.

The microbial payload can be launched as swarms of small microbial capsules, or the capsules can be bundled and protected through the interstellar journey to the targets where they will be dispersed by collisions with dust. The panspermia capsules will mix with the dust and condense with it into frozen and shielding in asteroids, comets and interplanetary dust particles. We can predict the conditions of these objects, because the outer zones of all solar nebulae should be similar, with temperatures $<50 \mathrm{~K}$, and contain ice, dust and organics similar to our early solar nebula.

Panspermia payloads to accretion zones or interstellar clouds will be captured in the dust and accreting asteroids and comets solar nebulae, similar to possible early life in asteroids in our Solar System during aqueous alteration [28,41-46]. The prospects for microbial life that was seeded into or arose locally in solar nebulae [42], are discussed below.

Some of the microbial capsules stored in asteroids and comets will be delivered later by meteorites and interplanetary dust particles (IDPs) to planets that developed habitable conditions. If the capsules are mixed homogenously with the dust, the fraction of the capsules delivered to the planets will be equal to the fraction of total dust delivered to planets as IDP particles, i.e. $P_{\text {(delivery) }}=m_{\text {(delivered dust) }} / m_{\text {(total dust) }}$.

The probability $P_{\text {(planet) }}$ that a capsule originally launched to the cloud will be delivered to the planet is then given by Equation (7).

$P_{(\text {planet })}=P_{(\text {target })} P_{(\text {delivery })}=\frac{P_{(\text {target })} m_{(\text {delivered dust })}}{m_{(\text {total dust })}}$

Tab. 3 The probability that panspermia missions will arrive at their target zones, and probability for eventual capture of one microbial capsule by a planet ${ }^{\mathrm{a}}$.

\begin{tabular}{|c|c|c|c|c|c|c|}
\hline & Distance (ly) & $\begin{array}{c}\text { Radius of target } \\
\text { area (au) }\end{array}$ & $\begin{array}{l}\text { Uncertainty of } \\
\text { target position } \\
\text { (au) }\end{array}$ & $\begin{array}{l}\text { Probability of } \\
\text { arriving in the } \\
\text { target zoneb }\end{array}$ & $\begin{array}{l}\text { Probability of } \\
\text { capture at a } \\
\text { target planet }\end{array}$ & $\begin{array}{l}\text { Biomass required } \\
\text { for probable } \\
\text { success }(\mathrm{kg})^{\mathrm{c}}\end{array}$ \\
\hline \multicolumn{7}{|c|}{ Nearby stars with accretion disks } \\
\hline Alpha PsA (Fomalhaut) & 22.6 & 3.2 & 3.1 & $>1^{\mathrm{b}}$ & $1.1 \times 10^{-5}$ & $1.0 \times 10^{-3}$ \\
\hline Beta Pictorius & 52.8 & 8.7 & 17 & 0.3 & $2.5 \times 10^{-6}$ & $4.4 \times 10^{-3}$ \\
\hline \multicolumn{7}{|c|}{ Rho Ophiuchus star-forming cloud } \\
\hline Dense fragment & 520 & 200000 & 1600 & $>1^{\mathrm{b}}$ & $1 \times 10^{-16}$ & $1.1 \times 10^{8}$ \\
\hline Protostellar condensation & 520 & 2000 & 80 & $>1^{\mathrm{b}}$ & $1 \times 10^{-13}$ & $1.1 \times 10^{5}$ \\
\hline Early accretion disk & 520 & 100 & 80 & 0.004 & $3.9 \times 10^{-14}$ & $2.8 \times 10^{5}$ \\
\hline Planetary feed zone & 520 & 3.5 & 80 & 0.000006 & $4.9 \times 10^{-11}$ & $2.2 \times 10^{2}$ \\
\hline
\end{tabular}

${ }^{a}$ Mission velocity $0.0005 c$ and parameters as described in [25]. ${ }^{b}$ Probabilities greater than unity mean that arrival or capture is virtually certain. ${ }^{c}$ Assuming the capture of 100 capsules with 0.11 microgram of microorganisms each, i.e., a total captured biomass of 11 micrograms. The required biomass is therefore given by biomass $=10^{-8} / P_{\text {capture }}$ kilograms [25]. 
If the probability for a launched capsule to reach a target planet is small, then sufficiently large numbers of capsules should be launched to assure a probability of landing on a planet, as given by Equation (8) will be near unity.

$$
n_{\text {(capsules) }}=\frac{1}{P_{(\text {planet })}}=\frac{m_{(\text {total dust })}}{P_{(\text {target })}} m_{(\text {delivered dust })}
$$

Penetration to target zones with various densities within the cloud can be achieved by choosing the mass of the vehicles, since heavier projectiles can penetrate into increasingly dense zones. Each capsule can be placed in a miniature spherical reflective film solar sail that does not need attitude control.

The above factors affect the probability that the microbial capsules will reach young planets. If the probability is small, more capsules with larger biomass need to be launched to increase the probability of success. The biomass required for successful missions in Tab. 3 was derived by these considerations.

These directed panspermia programs can be realized using current-level technologies, and may be easy to implement when a space infrastructure makes launch costs affordable. Even now, with present launch costs of $\$ 10000 / \mathrm{kg}$, a few hundred tons of microbial biomass with launch costs of about $\$ 1$ billion can seed dozens of new solar systems with our family of organic life to last there for eons.

\section{Biological and ecological challenges for directed panspermia}

Biological adaptation is key to life in space. The planted biota need to survive, procreate, grow and evolve in space habitats.

Fortunately, extremophile microorganisms can survive in a wide range of environments, from anaerobic to oxygenrich conditions, from below $0^{\circ} \mathrm{C}$ to over $140^{\circ} \mathrm{C}$, from low pressures at high altitudes to high pressures in deep seas, from basic solutions at high $\mathrm{pH}$ to concentrated sulfuric acid, from fresh water to concentrated brine, and also under intense radiation $[47,48]$.

Further, at currently achievable speeds of $0.0001 c$, the microorganisms need to survive cryptobiosis states for transit times up to 100000 years or more, which may be possible at interstellar temperatures of a few $\mathrm{K}$ in high vacuum. Faster transit times using more advanced interstellar propulsion methods are desirable [20].

Adapting life to such diverse extreme environments can involve fundamental biological changes, down to the basic molecular levels of DNA/protein life. These transformations need to be rapid if new environments are settled rapidly.

The microbial payloads can contain natural and bioengineered species with various tolerances, including designed microorganisms with combined multiple tolerances, hardy cyanobacterium akinetes, bacterial endospores that can survive interstellar travel without nutrients, and species resistant to UV radiation, desiccation in vacuum, freezing or high temperatures, and chemicals. It is a challenge to bioengineer space-adapted microorganisms with combinations of these various tolerances.

As noted above, sustainable seeded ecosystems require both photosynthetic autotrophs and biodegrading heterotrophs. Autotrophs are needed to synthesize complex biomolecules, and heterotrophs such as fungi are needed to degrade and recycle biomass. The autotrophs could be chemotrophs but preferably photosynthetic organisms such as algae that can propagate the chlorophyll-based apparatus and the genetic mechanisms to synthesize them. The heterotrophs may include fungi in lichens that are hardy colonizers. The combination of autotrophs and heterotrophs can form self-sustaining ecosystems, and facilitate evolution by predator/pray pressures.

Microbial ecosystems can fulfil the basic objective to continue DNA/protein life. However, as humans, we may also want to induce evolution toward conscious intelligent life. For this purpose we can include, along with the first colonizer microorganisms, hardy multicellular organisms such as rotifer cysts. Rotifers have the basic body-plans of higher organisms with differentiated organs and an animallike, but not insect-like, body-plan (Fig. 8). This will bypass a bottleneck to the evolution of multicellular organisms that took eons to develop on Earth. Rotifer eggs and cysts can possibly survive long interstellar journeys when deeply frozen. Tardigrades could also survive space travel [49].

Life in space, including directed panspermia, will encounter diverse environments that pose basic challenges for biology. Fortunately, the laws of physics and chemistry that underlie biology allow a broad range of biological transformations. The only constraints are the tests of survival that will always challenge life, whether produced naturally or by conscious designs.

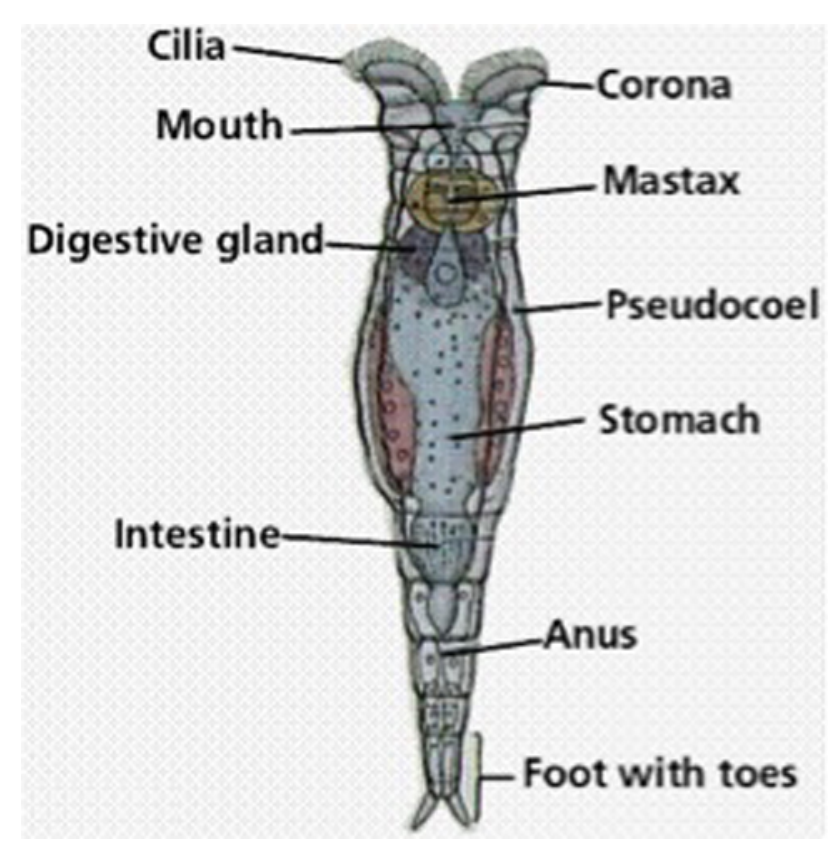

Fig. 8 Rotifers with animal body plan can jump-start higher evolution.

\section{Prospects of directed panspermia}

The technologies for directed panspermia are advancing: solar sailing and interstellar propulsion, precise astrometry, search for extrasolar planets, natural and bioengineered extremophile microorganisms. As a space infrastructure 
develops to fulfill human needs, launching directed panspermia missions will become possible for motivated individuals, small groups and organizations.

This program may affect the future of life, and possibly that of the physical galaxy, more than any other human activity. These fateful prospects, that affect the heritage of all, should be discussed publicly in the next decades while the technologies are advancing. The immense potentials of future life can contribute ethical incentives for space development.

\section{Directed panspermia and panbiotic ethics}

A concern about directed panspermia is that it may interfere with local life. This probability can be minimized by seeding newly forming young solar systems where local life, especially advanced life, could not have developed yet. In any case, we may at best seed a few dozen new solar systems, which would secure life but leave the vast majority of stars untouched for future exploration.

The only life known presently is our organic DNA/ protein life on Earth. The existence of life outside our Solar System is unproven and its probability cannot be quantified. If our microbial messengers encounter other branches of DNA/protein life, they may be both enriched by a genetic exchange. If they meet other, different life-forms, they will not interfere with it.

If we are alone, all life in the universe will end with end of the Sun, and the immense potentials of life in space will be lost. We can secure life now to make sure that this does not happen. It would seem irresponsible to abandon the only life that we know to exist to a certain end, for concerns about extraterrestrial life that may or may not exist.

A full scope of these panbiotic ethics can be derived from life-centered principles as discussed below $[4,38]$. They can secure the propagation of life if these ethics themselves are always propagated.

\section{Cosmo-ecology and the ultimate future}

\section{An overview}

Once life is established in space, what is its cosmological outlook?

Life can develop in many new directions given cosmological resources and timescales. The future forms of life are unpredictable, but its amounts will be defined by the available resources. Cosmo-ecology quantifies these potentials, and life-centered panbiotic ethics aims to realize them.

Cosmo-ecology can estimate the possible amounts of future life in the galaxy and in the universe [in units of BIOTA $_{\text {int }}$ (kg-years)]. Starting with the Solar System, life can survive for $10^{20}$ years about the red giant Sun and then about the subsequent white dwarf Sun, and likewise about other similar stars $[4,7,50]$.

Quantitatively, the amount of life on Earth in the last billion years has been on the order of $10^{15} \mathrm{~kg} \times 10^{9}$ years $=10^{24}$ kg-years (assuming a constant biomass of $10^{15} \mathrm{~kg}$ during this period). In comparison, the energy of red and white dwarf stars can sustain $10^{46} \mathrm{~kg}$-years of BIOTA ${ }_{\text {int }}$ during trillions of eons in the galaxy $[7,50]$. The potential future of life is immensely greater than its past.
At the theoretical limits all matter could be incorporated into biomass, and then converted gradually into energy to power this biomass. This yields the maximum possible amounts of life in the universe. However, the actual longterm future depends on dark matter and dark energy that have not been characterized yet. The 14 billion years since the Big Bang were just a brief moment in the long-term evolution of these forces, and predicting their future, and with it the future of life and the universe, may be possible only after further observation for thousands of future eons. Speculatively, life may continue indefinitely also in other universes [51], but they are unobservable by definition.

In the known universe life has immense potentials. To realize these potentials, we can preserve, continue, secure and expand life in space. Our descendants will be here then to understand nature more deeply, and reach for eternity.

\section{Future life in the Solar System}

The following discussions assume an efficiency of $10 \%$ for collecting and converting stellar radiation to biological energy, and a power use of $10 \mathrm{~W} / \mathrm{kg}$ by metabolically active biomass, altogether $100 \mathrm{~W}$ of stellar power $/ \mathrm{kg}$ biomass. The energy from stellar sources in various periods is estimated [50], and future technologies may capture them all in Dyson spheres [27].

The contribution of energy sources to time-integrated biomass BIOTA int (kg-years) in an ecosystem is given by Equation (9), where $L_{\text {source }}(\mathrm{W})$ is the luminosity of the source (energy output/time), $C_{\text {eff }}$ is the efficiency of collection and conversion of radiation to biological energy, $t_{\text {source }}$ (year) the life-time of the energy source, $n_{\text {source }}$ is the number of similar energy sources in the ecosystem (here, stars in the galaxy) and $P_{\text {biomass }}$ is the power demand of the biomass [W/ (kg biomass)].

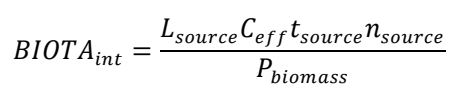

After the current main sequence phase, the Sun will become a red giant and then a white dwarf star following the patterns of main sequence stars [50]. Populations can survive these transitions by moving further or closer to the Sun as its luminosity changes. Note that photosynthetic plants such as asparagus can grow under a range of light intensities, down to solar irradiation at 9 astronomical units (AU) at Saturn [9], and maybe out to $300 \mathrm{AU}$ [52]. The $3.8 \times 10^{26} \mathrm{~W}$ luminosity of the main sequence Sun could then sustain about $4 \times 10^{24}$ $\mathrm{kg}$ biomass for a total of $10^{10}$ years, yielding BIOTA $_{\mathrm{int}}=4$ $\times 10^{34} \mathrm{~kg}$-years, and $10^{11}$ similar main sequence stars in the galaxy can contribute BIOTA $A_{\text {int }}$ of $4 \times 10^{45} \mathrm{~kg}$-years to the life in the galaxy [7]. Interestingly, biological resource elements from the total mass of the asteroids and comets can yield a similar time-integrated biomass.

After the Sun becomes a white dwarf, its $10^{15} \mathrm{~W}$ luminosity can sustain $10^{13} \mathrm{~kg}$ biomass, and the luminosity of the estimated $10^{12}$ white dwarfs can sustain $10^{25} \mathrm{~kg}$ biomass in the galaxy for $10^{20}$ years, yielding $10^{45} \mathrm{~kg}$-years [7,50].

Tab. 4 shows the estimated contributions of the various types of stars to the total BIOTA $A_{\text {int }}$ in the galaxy during its habitable lifetime. The eventual red giant stages of stars contribute $6 \times 10^{44} \mathrm{~kg}$-years of BIOTA $_{\text {int }}$ in the galaxy, considering 
Tab. 4 Life supported by the principal resources in future periods of cosmology ${ }^{\mathrm{a}}$.

\begin{tabular}{|c|c|c|c|c|c|c|c|}
\hline Location & $\begin{array}{l}\text { Materials, mass } \\
(\mathrm{kg})\end{array}$ & Power (W) & $\begin{array}{l}\text { No. in the } \\
\text { galaxy }\end{array}$ & $\begin{array}{l}\text { Future lifetime } \\
\text { (y) }\end{array}$ & Biomass $(\mathbf{k g})^{\mathrm{a}, \mathrm{b}}$ & $\begin{array}{c}\text { BIOTA }_{\mathrm{int}}(\mathrm{kg}- \\
\mathrm{y})^{\mathrm{a}, \mathrm{b}}\end{array}$ & $\begin{array}{c}B I O T A_{\text {int }} \text { in } \\
\text { galaxy }(\mathbf{k g}-\mathrm{y})^{\mathrm{a}}\end{array}$ \\
\hline Earth to present & & & & $4 \times 10^{9}$ & $10^{15 c}$ & $4 \times 10^{24 c}$ & \\
\hline Solar System & Asteroids, $10^{22}$ & $4 \times 10^{26}$ & $10^{11}$ & $5 \times 10^{9}$ & $\begin{array}{r}5 \times 10^{18 \mathrm{~d}} \\
\left(6 \times 10^{20}\right)^{\mathrm{e}}\end{array}$ & $\begin{array}{r}3 \times 10^{28 \mathrm{~d}} \\
\left(3 \times 10^{30}\right)^{\mathrm{e}}\end{array}$ & $\begin{array}{r}3 \times 10^{39 \mathrm{~d}} \\
\left(3 \times 10^{41}\right)^{\mathrm{e}}\end{array}$ \\
\hline Solar System & Comets, $10^{26}$ & $4 \times 10^{26}$ & $10^{11}$ & $5 \times 10^{9}$ & $\begin{array}{c}5 \times 10^{22 \mathrm{~d}} \\
\left(6 \times 10^{24}\right)^{\mathrm{e}}\end{array}$ & $\begin{array}{r}3 \times 10^{32 \mathrm{~d}} \\
\left(3 \times 10^{34}\right)^{\mathrm{e}}\end{array}$ & $\begin{array}{r}3 \times 10^{43 \mathrm{~d}} \\
\left(3 \times 10^{45}\right)^{\mathrm{e}}\end{array}$ \\
\hline Red giants & Comets, $10^{26}$ & $10^{30}$ & $10^{11}$ & $10^{9}$ & $6 \times 10^{24 \mathrm{e}}$ & $6 \times 10^{33 \mathrm{e}}$ & $6 \times 10^{44 \mathrm{e}}$ \\
\hline White dwarfs & Comets, $10^{26}$ & $10^{15}$ & $10^{12}$ & $10^{20}$ & $10^{13 \mathrm{f}}$ & $10^{33 \mathrm{~d}}$ & $10^{45}$ \\
\hline Red dwarfs & & $10^{23}$ & $10^{12}$ & $10^{13}$ & $10^{21 \mathrm{f}}$ & $10^{34}$ & $10^{46}$ \\
\hline Brown dwarfs & & $10^{20}$ & $10^{12}$ & $10^{10}$ & $10^{18 \mathrm{f}}$ & $10^{28}$ & $10^{39}$ \\
\hline Galaxy & Baryons, $10^{41}$ & $m c^{2} / t$ & & $10^{37 \mathrm{~g}}$ & $<10^{41}$ & & $10^{48 \mathrm{~h}}$ \\
\hline Universe & Baryons, $10^{52}$ & $m c^{2} / t$ & & $10^{37 \mathrm{~g}}$ & $<10^{52 \mathrm{i}}$ & & $10^{59 \mathrm{~h}, \mathrm{i}}$ \\
\hline
\end{tabular}

${ }^{a}$ The figures are order-of-magnitude estimates and the digits shown indicate the results of the calculations but don't imply this degree of accuracy. ${ }^{\mathrm{b}}$ Per solar system. ${ }^{\mathrm{c}}$ Assuming the estimated present $10^{15} \mathrm{~kg}$ biomass [10] for the past $4 \times 10^{9}$ years, as an upper limit. ${ }^{\mathrm{d}}$ Biomass obtained using water-soluble elements in asteroids or comets, respectively, based on $\mathrm{N}$ as the limiting nutrient. ${ }^{\mathrm{e}}$ Biomass obtained using total elemental contents of asteroids or comets, respectively, based on $\mathrm{N}$ as the limiting nutrient. ${ }^{\mathrm{f}}$ Biomass based on power supply of $100 \mathrm{~W} / \mathrm{kg}$ as the limiting factor. ${ }^{\mathrm{g}}$ Estimated proton decay time [50]. ${ }^{\mathrm{h}}$ Based on the dissipation of mass as bioavalaible energy. ${ }^{\mathrm{i}}$ Amount in the universe [7].

the limiting factor to be nitrogen in the resource asteroids and comets $[7,8]$.

The limiting factor for the white, red and brown dwarf stars is assumed to be energy, and they can contribute $10^{45}$, $10^{46}$, and $10^{39} \mathrm{~kg}$-years of time-integrated biomass, $B I O T A_{\text {int }}$ in the galaxy, respectively (Tab. 4). Interestingly, all the main long-lived types of stars except brown dwarfs contribute similar integrated energy output (luminosity $\times$ lifetime $\times$ $\left.n_{\text {galaxy }}\right)$ in the galaxy. Correspondingly, they can contribute similar BIOTA $A_{\text {int }}$ of about $10^{45}-10^{46} \mathrm{~kg}$-years to the potential maximum amount of life in the galaxy that can be based on stellar energy.

\section{Life in solar nebulae}

Panspermia payloads to accretion zones or interstellar clouds will be captured in solar nebulae, or microbial life may arise in or be transported naturally to asteroids during aqueous alteration $[28,41,42]$. Tests of meteorites showed that microorganisms can be sustained by the nutrients in these asteroid solutions $[8,9]$.

These solutions in cavities and pores of asteroids could contain concentrated, several mol/l organic solutions as our meteorite experiments showed. The organics there can be activated by catalytic metal ions, minerals and clays $[8,9]$, and by the radioactive decay of rocks, while trapped for $10^{6}-10^{7}$ years of aqueous alteration at temperatures from 0 to $>140 \mathrm{C}$ in reducing conditions under high pressure hydrogen, containing ammonia and methane. Organics in carbonaceous chondrite meteorites show that complex molecules including amino acids and nucleic bases form under these conditions.

Quantitatively, porosities of $15-25 \%$ of carbonaceous chondrites [43] allow asteroids to contain large volumes of nutrient pore solutions. In $4-6 \times 10^{6}$ years of aqueous alteration [44], organic reactions in solutions with half-lives of seconds or shorter can allow stepwise synthetic reactions of thousands up to millions of steps, to produce the complex molecules required to form a microorganism.

Bioavailable nutrients in a large asteroid can then yield enough microbial populations to seed the $10^{22} \mathrm{~kg}$ of asteroids if the asteroid is fragmented and scattered. The microorganisms may also land on hyperbolic comets that carry them to interstellar space. Nutrients in a meteorite can yield enough microorganisms to colonize a planet, and nutrients in an asteroid belt can yield enough microorganisms to seed the galaxy.

For example, a $10 \mathrm{~km}$ radius, $4 \times 10^{12} \mathrm{~m}^{3}$ and $10^{16} \mathrm{~kg}$ asteroid with $20 \%$ porosity would contain $8 \times 10^{14} 1$ pore solutions with $10^{13} \mathrm{~kg}$ organic $\mathrm{C}$ (based on $1 \mathrm{~g} / \mathrm{kg}$ soluble $\mathrm{C}$ ). The total $10^{22} \mathrm{~kg}$ asteroids would contain $10^{19} \mathrm{~kg}$ dissolved organic $\mathrm{C}$, in $0.01 \mathrm{~kg} / \mathrm{l}$ (about $0.1 \mathrm{~mol} / \mathrm{l}$ ) solutions. During a million years of aqueous alteration, and with reaction half-lives of seconds to years, these solutions allow multi-step chemical synthesis of $10^{6}-10^{13}$ steps to build up complex proteins or RNA leading to microbial life. Similar processes can occur later in the pores of carbonaceous chondrite meteorites landed in water on planets $[8,9]$.

If microbial life starts in an asteroid, the organics may become sequestered in microbial biomass or freeze, stopping evolution. However, these microorganisms can start evolution later when delivered by meteorites to planets.

To examine this mechanism quantitatively, a C-type asteroid with phosphorus as the limiting nutrient yield about $10^{-4} \mathrm{~kg}$ biomass $/ \mathrm{kg}$ resource material. A $10 \mathrm{~km}, 10^{16} \mathrm{~kg}$ early asteroid could then yield a bacterial biomass of $10^{12} \mathrm{~kg}$ with $10^{27}$ microorganisms. With doubling time of one day, a single $10^{-15} \mathrm{~kg}$ inoculating microorganism could develop into this $10^{12} \mathrm{~kg}$ population of $10^{27}$ microorganisms in 90 days.

This microbial biomass would sequester the organics of the asteroid, and without recycling this would stop growth and evolution. However, if the $10^{27}$ microorganisms in the parent asteroid scatter in the asteroid belt, they can provide 
$10^{5}$ inoculant microorganisms per $\mathrm{kg}$ asteroid (e.g., $10^{21}$ microorganisms for a $10 \mathrm{~km}, 10^{16} \mathrm{~kg}$ asteroid) to colonize all the $10^{22} \mathrm{~kg}$ asteroid population in the early Solar System. Similarly, microorganisms in a $10 \mathrm{~kg}$ meteorite with a CM2 Murchison-like composition could reach a biomass of $1 \mathrm{~g}$ with a population of $10^{12}$ microorganisms, sufficient to seed a planet. Further, soluble materials in the $10^{22} \mathrm{~kg}$ asteroids can yield a microbial biomass up to $10^{18} \mathrm{~kg}$ comprising $10^{33}$ microorganisms that could seed $10^{11}$ solar systems in the galaxy each with $10^{22}$ microorganisms.

Beyond asteroids, comets may also host and distribute microorganisms. Subsurface ice may melt close to perihelion [45], and its organics can yield a solution similar to those in meteorites, that support algae and fungi $[8,9]$.

Heterotrophs, chemotrophs, lithophiles and psychrophiles could multiply in these solutions, then eject with the coma in dust particles that protect them in space. They could be also transported through interstellar space by hyperbolic comets. These mechanisms were considered for natural panspermia [46], and for directed panspermia that could also use genetically engineered microorganisms suited to these conditions $[25,42,46,47]$.

In summary, based on measured nutrients in meteorites, nutrients in a meteorite can produce enough microorganisms to seed a planet; nutrients in an asteroid can produce enough microorganisms to seed the asteroid belt; and nutrients in one asteroid belt can produce enough microorganisms to seed all the solar systems in the galaxy.

\section{Life in a finite universe}

The theoretical upper limits of the amounts of life in the universe would be realized if all baryonic matter is converted to biomass, and a small fraction of the biomass $\left(3.5 \times 10^{-8} \mathrm{y}^{-1}\right)$ is then converted relativistically gradually to energy to sustain the biomass.

Assume that the power requirement of biomass is $P_{\text {biomass }}$ $\left(\mathrm{J} \mathrm{s}^{-1} \mathrm{~kg}^{-1}\right)$, and the energy yield factor of converting biomass to energy is $E_{\text {yield,biomass }}=E_{\text {biomass,released }} / m_{\text {biomass,converted }}=c^{2}$. If biomass is converted to energy at the rate needed to power the remaining biomass, then the biomass decays exponentially at the rate given by Equation (10).

$$
\left(-d m_{\text {biomass }} / d t\right) E_{\text {yeld,biomass }}=P_{\text {biomass }} m_{\text {biomass }}
$$
(11):

The remaining biomass after time $t$ is given by Equation

$$
m_{\text {biomass }, t}=m_{\text {biomass }, 0}=\exp \left(-\left(P_{\text {biomass }} / E_{\text {yield,biomass }}\right) t\right)
$$

The maximum energy can be obtained by converting mass to energy according to the relativistic relation $E=m c^{2}$. In this case $E_{\text {yield,biomass }}=c^{2}$, and assuming a power need of $P_{\text {bi }}$ $=100 \mathrm{~W} / \mathrm{kg}$ biomass, the decay rate of the biomass is $k_{\text {use }}=$ $100 /\left(3 \times 10^{8}\right)^{2}=1.1 \times 10^{-15} \mathrm{~s}^{-1}=3.5 \times 10^{-8} \mathrm{y}^{-1}$.

The amount of baryonic matter that could be converted to biomass in the galaxy is on the order of $10^{41} \mathrm{~kg}$. If a fraction of it was converted to energy at the rate shown in Equation (10), there would be enough biomass left for one $50 \mathrm{~kg}$ human in the galaxy after 2.6 billion years, and in the universe, after 3.3 billion years. The last remaining life, a single bacterium of $10^{-15} \mathrm{~kg}$, would be left after 3.7 billion years in the galaxy, and after 4.4 billion years in the universe. The total timeintegrated life in the galaxy would be $10^{48} \mathrm{~kg}$-years and in the universe $10^{59} \mathrm{~kg}$-years. The data in last rows in Tab. 4 were derived according to these considerations.

Although these amounts of life are immense, life would become extinct rapidly compared with the estimated ultimate decay of baryonic matter in $10^{37}$ years [50] as the biomass is converted to the energy needed to sustain it. In order to extend life throughout these possible $10^{37}$ years, the $10^{41} \mathrm{~kg}$ baryonic matter in the galaxy could be converted to biomass and then to energy more slowly, sustaining a steady-state biomass of $3 \times 10^{11} \mathrm{~kg}$ throughout this time, possibly as $10^{10}$ humans. The respective biomass and populations in the universe are larger than in the galaxy by a factor of $10^{11}$ (galaxies in the universe). Life in the galaxy and the universe would then last for $10^{37}$ (ten trillion trillion trillion) years through the habitable lifetime of the galaxy.

Future life is finite in a finite universe, while in an everexpanding universe, intelligent existence (not organic life) may be extended indefinitely at an ever slowing pace [5]. The above calculations and results are theoretical upper limits that are permitted by physical law. These potential amounts of life are immense, but finite.

If life is finite, should we propagate it? Even a small fraction of the potential time-integrated biomass can secure biological life for all foreseeable time. Biotic ethics recommend to realize as much of this potential life as possible. This will allow our remote descendants to observe nature more deeply, and use it to best serve life.

\section{Life-centered astroethics}

The future of life in space depends on human actions that are governed by our philosophies and ethics. These human activities are already transforming the Earth, and in the future they can affect the Solar System and the galaxy. In this manner, ethical principles can become physical forces.

These powerful ethics can be formulated on rational science-based principles. Such a fundamental principle is our identity as living beings, united with all other known organic DNA/protein life in the complex cellular machinery that underlies all biology. All of our organic cellular life share the complex structures and mechanisms of genetics, metabolism, enzyme catalysis, membrane transport, and ATP-based energy use. These complex biological structures and processes are special in Nature, because the laws of physics just narrowly allow biology to exist.

Self-propagation is also unique and common to all life. Our basic unity with all self-propagating life therefore implies a human purpose to protect, propagate and expand life. This purpose can define the basic values of life-centered biotic ethics: acts that support life are good and acts that destroy life are evil. Most cultures observe these principles.

The human purpose to propagate life is best secured in space. Once established in many worlds, the basic patterns of life can be secure through eons. The cosmological scales of time, space and resources allow life to realize the full potentials that biology permits. 
Expansion in space implies a technological society that can also self-destruct by violence, biological misengineering, or by replacing biological life with robots. Therefore in a self-fulfilling future, survival must be pursued deliberately to secure survival. This implies control by DNA/protein organic brains that have a vested self-interest to propagate biological life.

These life-centered ethics can be applied in space as panbiotic ethics that aim to secure and expand life. Human settlement of the Solar System serves this objective if all humans act in unison for the future. Directed panspermia can open even larger, galactic scale prospects for life, and it can be achieved more readily from a future space infrastructure, by groups or even individuals motivated by life-centered panbiotic ethics [4,23-25,38].

\section{Conclusions}

Expansion in space can open immense potentials for the growth of humankind and life. In turn, biological adaptation and life-centered biotic ethics are keys to this expansion $[4,32,38,39,47,52-56]$. To examine these potentials scientifically, it is necessary to quantify, based on experimental data, the potential scope of life in the Solar System and in the galaxy.

To this effect, experimental astroecology investigates the relationship between life and space resources, with the amount of life in an ecosystem measured as timeintegrated biomass (kg-years). In these studies, meteorite models of asteroid materials showed that bacteria, algae and plants can grow on asteroid/meteorite organics and electrolytes, with fertilities similar to productive agricultural soils $[4,8,9,26,57-61]$. Based on the measured soluble nutrient contents, asteroid resources can yield a biomass of $10^{18} \mathrm{~kg}$ in the Solar System. Extrapolating to a lifetime of $10^{10}$ years and to $10^{11}$ stars in the galaxy, similar resources could support a time-integrated biomass on the order of $10^{39}$ kg-years during the habitable lifetime of the galaxy, vastly greater than the $10^{24} \mathrm{~kg}$-years of life on Earth to date. On cosmological scales, energy from white and red dwarf stars can sustain life for $10^{20}$ (a hundred billion billion) years, yielding a time-integrated biomass of $10^{46} \mathrm{~kg}$-years in the galaxy and $10^{57} \mathrm{~kg}$-years in the universe.

This potential future life can be accessed with current-level technology. Human populations in the Solar System can use asteroid resources to achieve a population of $10^{14}$ (a hundred trillion) humans and their supporting biota. Considering a $1 \%$ per year irreversible waste, the Solar System can sustain $10^{7}$ (ten million) humans and their supporting biomass of $10^{11} \mathrm{~kg}$ for billions of years.

\section{Acknowledgments}

I thank the NASA/NSF "Antarctic meteorite program" for samples studied in the source papers. This work was not subject to external funding.

\section{Competing interests}

No competing interests have been declared.
These space-based human populations are vital for securing and expanding life in space. This is especially critical if we are alone $[4,23-25,62,63]$. To assess extra-Solar-System life, it is necessary to estimate the probability that life arises in favorable ecosystems. This probability is hard to estimate, because the vital functions of even the first viable cell would require complex processes subject to specific chemical and physical parameters, and also a sustaining early environment with restricted viable parameters $[6,28,41,64]$. These parameters can have many possible combinations, that cannot be tested experimentally, and only a few can support life. Therefore we may not be able to quantify the probability that life arises even in favorable environments. This leaves it possible that we are alone, and the future of life is in our hands.

We can secure this future by expansion in space. Human settlement in the Solar System can serve this objective, if all humans act in unison for the future. Directed panspermia can achieve even larger, galactic scale prospects for life, and it can be implemented more easily from a space infrastructure by groups or individuals motivated by life-centered panbiotic ethics.

This panbiotic program can allow DNA/protein organic life to access galactic scale resources, by seeding new solar systems where any local life could not have arisen yet. The payloads can include extremophiles suited to diverse environments, including autotrophs and heterotrophs to establish self-sustaining recycling ecosystems. Small hardy multicellular organisms can be also included, to jump-start higher evolution toward intelligent species that may further expand life in space.

With our powerful technologies our designs can become self-fulfilling, and to secure survival, it must be then sought purposefully. The guiding ethics can be based on scientific insights: our basic biological unity with all cellular, organic DNA/protein life; the special place of complex life in nature, that precisely allows biology to exist; and the shared drive of all life for self-propagation. Our unity with all life then implies life-centered biotic ethics that aim to propagate life, applied in space as panbiotic ethics to expand life. To secure survival in a self-designed future, these ethics themselves must be also always propagated.

With these life-centered ethics, the cosmological spans of space, time and resources can support an immense future. We can plant that future by seeding new worlds with organic DNA/protein life. A great diversity of life can then emerge, some intelligent who understand nature more deeply and seek to extend life indefinitely. In securing that future for life, our human endeavors can fulfill a cosmic purpose.

\section{References}

1. Rynin NA. Interplanetary flight and communication. Volume 3, No. 7: K. E. Tsiolkovskii, life, writings, and rockets. Washington, DC: Israel Program for Scientific Translations; 1971.

2. Dyson FJ. Disturbing the universe. New York, NY: Harper and Row; 1979. 
3. O'Neill GK. The colonization of space. Phys Today. 1974;27(9):32-38.

4. Mautner MN. Seeding the universe with life: securing our cosmological future: galactic ecology, astroethics and directed panspermia. Washington, DC: Legacy Books; 2000.

5. Dyson FJ. Time without end: biology and physics in an open universe. Rev Mod Phys. 1979;5:447-460.

6. Purves WK, Orians GH, Sadava D, Heller HC. Life, the science of biology. Sunderland, MA: Sinauer Associates and W.H. Freeman; 2001.

7. Mautner MN. Life in the cosmological future - resources, biomass and populations. J Br Interplanet Soc. 2005;58:167-180.

8. Mautner MN. Planetary resources and astroecology. planetary microcosm models of asteroid and meteorite interiors: electrolyte solutions and microbial growth - implications for space populations and panspermia. Astrobiology. 2002;2(1):59-76. http://dx.doi. org/10.1089/153110702753621349

9. Mautner M. Planetary bioresources and astroecology. 1. Planetary microcosm bioassays of Martian and carbonaceous chondrite materials: nutrients, electrolyte solutions, and algal and plant responses. Icarus. 2002;158(1):72-86. http://dx.doi.org/10.1006/icar.2002.6841

10. Bowen HJM. Trace elements in biochemistry. New York, NY: Academic Press; 1966.

11. Bolonkin AA. Making asteroids habitable. In: Badescu V, editor. Asteroids. Berlin: Springer; 2013. p. 561-580. http://dx.doi. org/10.1007/978-3-642-39244-3_24

12. McKay CP, Toon OB, Kasting JF. Making Mars habitable. Nature. 1991;352(6335):489-496. http://dx.doi.org/10.1038/352489a0

13. Fogg MJ. Terraforming: a review for environmentalists. Environmentalist. 1993;13(1):7-17. http://dx.doi.org/10.1007/BF01905499

14. Mautner MN, Sinaj S. Water-extractable and exchangeable phosphate in Martian and carbonaceous chondrite meteorites and in planetary soil analogues. Geochim Cosmochim Acta. 2002;66:3161-3174.

15. Mauldin JH. Prospects for interstellar travel. San Diego, CA: Univelt; 1992. (Science and technology series; vol 80).

16. Mallove EF. The starflight handbook: a pioneer's guide to interstellar travel. New York, NY: Wiley; 1989.

17. Glaser PE. Power from the Sun: its future. Science. 1968;162(3856):857861. http://dx.doi.org/10.1126/science.162.3856.857

18. Mautner MN. A space-based solar screen against climatic warming. J Br Interplanet Soc. 1991;44:135-138.

19. Bewick R, Sanchez JP, McInnes CR. Gravitationally bound geoengineering dust shade at the inner Lagrange point. Adv Space Res. 2012;50(10):1405-1410. http://dx.doi.org/10.1016/j.asr.2012.07.008

20. Matloff GL, Johnson L, Bangs C. Living off the land in space: green roads to the cosmos. New York, NY: Springer; 2001.

21. Sauser B. A Moon-based telescope [Internet]. MIT Technology Review. 2008 [cited 2014 Dec 20]; Available from: http://www.technologyreview.com/news/410253/a-moon-based-telescope/

22. Mautner MN. Space-based genetic cryoconservation of endangered species. J Br Interplanet Soc. 1996;49:319-320.

23. Mautner MN, Matloff GL. Directed panspermia - a technical and ethical evaluation of seeding other solar systems. J Br Interplanet Soc. 1979;48:435-440.

24. Mautner MN, Matloff GL. Directed panspermia. 2. Technological advances toward seeding other solar systems, and the foundation of panbiotic ethics. J Br Interplanet Soc. 1995;48:435-440.

25. Mautner MN. Directed panspermia. 3. Strategies and motivation for seeding star-forming clouds. J Br Interplanet Soc. 1997;50:93-102.

26. Mautner MN. In situ biological resources: soluble nutrients and electrolytes in carbonaceous asteroids/meteorites. Implications for astroecology and human space populations. Planet Space Sci. 2014;104:234-243. http://dx.doi.org/10.1016/j.pss.2014.10.001

27. Dyson FJ. Search for artificial stellar sources of infrared radiation. Science. 1960;131(3414):1667-1668. http://dx.doi.org/10.1126/ science.131.3414.1667

28. Chyba CF, McDonald GD. The origin of life in the Solar System: current issues. Annu Rev Earth Planet Sci. 1995;23(1):215-249. http:// dx.doi.org/10.1146/annurev.ea.23.050195.001243
29. Lynch SR, Liu H, Gao J, Kool ET. Toward a designed, functioning genetic system with expanded-size base pairs: solution structure of the 8-base xDNA double helix. J Am Chem Soc. 2006;128(45):1470414711. http://dx.doi.org/10.1021/ja065606n

30. Pinheiro VB, Taylor AI, Cozens C, Abramov M, Renders M, Zhang $\mathrm{S}$, et al. Synthetic genetic polymers capable of heredity and evolution. Science. 2012;336(6079):341-344. http://dx.doi.org/10.1126/ science. 1217622

31. Herdman M, Janvier M, Rippka R, Stanier RY. Genome size of cyanobacteria. J Gen Microbiol. 1979;111(1):73-85. http://dx.doi. org/10.1099/00221287-111-1-73

32. Jacobsen JH. Genetic engineering of cyanobacteria [PhD thesis]. Copenhagen: University of Copenhagen; 2012.

33. Maccone C. The statistical Drake equation. Acta Astronaut. 2010;67(1112):1366-1383. http://dx.doi.org/10.1016/j.actaastro.2010.05.003

34. Haldane JBS. The origins of life. New Biol. 1954;16:12-27.

35. Shklovskii IS, Sagan C. Intelligent life in the Universe. San Francisco, CA: Holden-Day; 1996.

36. Crick FHC, Orgel LE. Directed panspermia. Icarus. 1973;19(3):341346. http://dx.doi.org/10.1016/0019-1035(73)90110-3

37. Zuckerman B. Space telescopes, interstellar probes and directed panspermia. J Br Interplanet Soc. 1981;34:367-370.

38. Mautner MN. Life-centered ethics, and the human future in space. Bioethics. 2009;23(8):433-440. http://dx.doi. org/10.1111/j.1467-8519.2008.00688.x

39. Makukov MA, shCherbak VI. Space ethics to test directed panspermia. Life Sci Space Res Amst. 2014;3:10-17. http://dx.doi.org/10.1016/j. 1ssr.2014.07.003

40. Mezger PG. The search for protostars using millimeter/submillimeter dust emission as a tracer. In: Burke BF, Rahe JH, Roettger EE, editors. Planetary systems: formation, evolution, and detection. Dordrecht: Springer; 1994. p. 197-214. http://dx.doi. org/10.1007/978-94-011-1154-6_22

41. Pizzarello $S$. The chemistry of life's origin: a carbonaceous meteorite perspective. Acc Chem Res. 2006;39(4):231-237. http://dx.doi. org/10.1021/ar050049f

42. Mautner MN, Ibrahim Y, El-Shall MS. Organic synthesis and potential microbiology in the Solar Nebula: are early Solar Systems nurseries for microorganisms? Int J Astrobiol. 2004;3(1 suppl):101.

43. Macke RJ. Survey of meteorite physical properties: density, porosity and magnetic susceptibility [PhD thesis]. Orlando, FL: University of Central Florida; 2010.

44. Guo W, Eiler JM. Temperatures of aqueous alteration and evidence for methane generation on the parent bodies of the $\mathrm{CM}$ chondrites. Geochim Cosmochim Acta. 2007;71(22):5565-5575. http://dx.doi. org/10.1016/j.gca.2007.07.029

45. Lewis JS. Physics and chemistry of the solar system. New York, NY: Academic Press; 1997.

46. Hoyle F. Lifecloud: the origin of life in the universe. London: J. M. Dent; 1978.

47. Montague M, McArthur GH, Cockell CS, Held J, Marshall W, Sherman LA, et al. The role of synthetic biology for in situ resource utilization (ISRU). Astrobiology. 2012;12(12):1135-1142. http:// dx.doi.org/10.1089/ast.2012.0829

48. Horikoshi K, Grant WD, editors. Extremophiles: microbial life in extreme environments. New York, NY: Wiley; 1998.

49. Jönsson KI, Rabbow E, Schill RO, Harms-Ringdahl M, Rettberg P. Tardigrades survive exposure to space in low Earth orbit. Curr Biol. 2008;18(17):R729-R731. http://dx.doi.org/10.1016/j.cub.2008.06.048

50. Adams F, Laughlin G. The five ages of the universe: inside the physics of eternity. New York, NY: Touchstone; 1999.

51. Bousso R, Susskind L. The multiverse interpretation of quantum mechanics. Phys Rev Part Fields. 2012;85(4). http://dx.doi.org/10.1103/ PhysRevD.85.045007

52. Raven JA, Kübler JE, Beardall J. Put out the light, and then put out the light. J Mar Biol Assoc UK. 2000;80(01):1-25.

53. Chyba C, Sagan C. Endogenous production, exogenous delivery 
and impact-shock synthesis of organic molecules: an inventory for the origins of life. Nature. 1992;355(6356):125-132. http://dx.doi. org $/ 10.1038 / 355125 \mathrm{a} 0$

54. Olsson-Francis K, de la Torre R, Towner MC, Cockell CS. Survival of akinetes (resting-state cells of cyanobacteria) in low earth orbit and simulated extraterrestrial conditions. Orig Life Evol Biosph. 2009;39(6):565-579. http://dx.doi.org/10.1007/s1 1084-009-9167-4

55. Hart MH. Interstellar migration, the biological revolution, and the future of the galaxy. In: Finney BR, Jones EM, editors. Interstellar migration and the human experience. Berkeley, CA: University of California Press; 1986. p. 278-291.

56. Fukuyama F. Our posthuman future: consequences of the biotechnology revolution. New York, NY: Farrar Straus \& Giroux; 2002.

57. Mautner MN, Leonard RL, Deamer DW. Meteorite organics in planetary environments: hydrothermal release, surface activity, and microbial utilization. Planet Space Sci. 1995;43(1-2):139-147.

58. Mautner MN, Conner AJ, Killham K, Deamer DW. Biological potential of extraterrestrial materials. 2. Microbial and plant responses to nutrients in the Murchison carbonaceous meteorite. Icarus. 1997;129:245-253
59. Mautner MN. Biological potential of extraterrestrial materials. I. Nutrients in carbonaceous meteorites, and effects on biological growth. Planet Space Sci. 1997;45(6):653-664. http://dx.doi.org/10.1016/ S0032-0633(97)00017-2

60. Kennedy J, Mautner MN, Barry B, Markwitz A. Microprobe analysis of brine shrimp grown on meteorite extracts. Nucl Instrum Methods Phys Res B. 2007;260(1):184-189. http://dx.doi.org/10.1016/j. nimb.2007.02.022

61. Marcano V, Matheus P, Cedeño C, Falcon N, Palacios-Prü E. Effects of non-carbonaceous meteoritic extracts on the germination, growth and chlorophyll content of edible plants. Planet Space Sci. 2005;53(12):1263-1279. http://dx.doi.org/10.1016/j.pss.2005.05.003

62. O’Neill GK. The high frontier. New York, NY: William Morrow; 1977.

63. Davies P. The eerie silence. Boston, MA: Houghton Mifflin Harcourt; 2010.

64. Yockey HP. Origin of life on Earth and Shannon's theory of communication. Comput Chem. 2000;24(1):105-123. http://dx.doi.org/10.1016/ S0097-8485(00)80010-8 